ISPUmath- $1 / 2006$

\title{
GARDNER'S DEFORMATIONS OF THE BOUSSINESQ EQUATIONS
}

\author{
ATALAY KARASU AND ARTHEMY V. KISELEV
}

\begin{abstract}
Using the algebraic method of Gardner's deformations for completely integrable systems, we construct the recurrence relations for densities of the Hamiltonians for the Boussinesq and the Kaup-Boussinesq equations. By extending the Magri schemes for these equations, we obtain new integrable systems adjoint with respect to the initial ones and describe their Hamiltonian structures and symmetry properties.
\end{abstract}

Introduction. In this paper we consider the most efficient way to prove the complete integrability of evolutionary systems. Namely, we apply the method of Gardner's deformations [1] -6] to the Boussinesq and Kaup-Boussinesq equations. By construction, the deformations consist of the (multi-)Hamiltonian scaling non-invariant parametric extensions $\mathcal{E}_{\varepsilon}$ of the original systems $\mathcal{E}_{0}$ and the parameter-dependent Miura transformations $\mathcal{E}_{\varepsilon} \rightarrow \mathcal{E}_{0}$. Inverting the Miura transformations from the new systems, one obtains the recurrence relations for the infinite sequences of densities of the Hamiltonian functionals. We improve the result of Kupershmidt in [3] by showing that the sequences of conserved densities for the Boussinesq equation satisfy two different recurrence relations simultaneously. We demonstrate that the extended equations must not necessarily interpolate (as it is assumed in [7]) between the equations $\mathcal{E}_{0}$ and the modified systems that provide the canonical factorizations for the higher Poisson structures [8]. The recurrence relations we obtain are inherited by the modified Boussinesq [9] and Kaup-Boussinesq [10] equations.

Next, we separate the flows at the deformation parameters and determine the adjoint Boussinesq equations whose Magri schemes are coupled with the non-extended ones. We show that the new systems are also integrable.

The examples discussed in this paper are essentially used in the general algebraic approach to the problem of Gardner's deformations and integrable extensions of the Magri schemes. This approach is based on the notion of coverings over PDE [11] and their parametric families constructed using the Frölicher-Nijenhuis bracket [12, 13, 14]. This will be the object of a subsequent publication.

The paper is organized as follows. In section 1 we obtain two Gardner's deformations for the Boussinesq equation using the dispersionless case [15] as the starting point. We specify the adjoint Boussinesq systems and discuss their integrability. In section 2 we construct the deformation of the Kaup-Boussinesq equation and describe the biHamiltonian adjoint system.

Date: March 21, 2006.

2000 Mathematics Subject Classification. 35Q53, 37K05, 37K10, 37K35. PACS 02.30.Ik.

Key words and phrases. Gardner's deformations, integrable extensions, Boussinesq equation, KaupBoussinesq equation. 


\section{The Boussinesq equation}

First we consider the Boussinesq equation with dispersion and dissipation, see [3],

$$
\mathcal{U}_{t}=\mathcal{V}_{x}+\alpha \mathcal{U}_{x x}, \quad \mathcal{V}_{t}=\mathcal{U}_{x x x}+\mathcal{U U}_{x}-\alpha \mathcal{V}_{x x}, \quad \alpha \in \mathbb{R}
$$

We note that for any $\alpha$ system (11) is transformed to the equation $\mathcal{U}_{t t}=\left(\mathcal{U}_{x x x} \cdot(1+\right.$ $\left.\alpha)+\mathcal{U U}_{x}\right)_{x}$, which is scaling-equivalent to the Boussinesq equation $u_{t t}=\left(u_{x x x}+u u_{x}\right)_{x}$ whenever $\alpha \neq-1$. Therefore for $\alpha \neq-1$ equation (II) is reduced to the Boussinesq equation

$$
u_{t}=v_{x}, \quad v_{t}=u_{x x x}+u u_{x} .
$$

In the sequel, we consider the problem of Gardner's deformation for system (2).

Next, we observe that a Gardner's deformation for (2) (and for the Kaup-Boussinesq equation (77) as well) is obtained from the deformation of the dispersionless reduction by adding higher order terms to the equations $\mathcal{E}_{\varepsilon}$ and to the Miura transformations $\mathcal{E}_{\varepsilon} \rightarrow \mathcal{E}_{0}$. Indeed, the zero order terms in the conserved densities, which are obtained recursively by inverting the Miura substitutions, originate from the relations for the dispersionless systems. The deformation for the dispersionless Boussinesq equation has been described in 15]; it corresponds to the case $\alpha=-1$ in (11). Using this result, we construct two deformations for (2) and get two distinct recurrence relations for both sequences of the conserved densities. Thus we improve the result of [3], where a recurrence relation for only one sequence was obtained. The adjoint Boussinesq equations that appear in the extended systems are the by-products of our reasonings.

Theorem 1. There are two deformations $\mathcal{E}_{\varepsilon}^{ \pm}$for the Boussinesq equation (2). Both of them are Hamiltonian w.r.t. the structure

$$
\left(\begin{array}{cc}
0 & D_{x} \\
D_{x} & 0
\end{array}\right)
$$

and the functionals with the densities

$$
H_{ \pm}(\varepsilon)=\frac{1}{6} \tilde{u}^{3}+\frac{1}{2} \tilde{v}^{2}+\varepsilon^{3} \cdot\left(\frac{1}{6} \tilde{v}^{3}+\frac{1}{2} \tilde{u}_{x}^{2} \tilde{v} \pm \frac{1}{2} \tilde{u}_{x} \tilde{v}^{2} \pm \frac{1}{6} \tilde{u}_{x}^{3}\right),
$$

respectively.

The deformed equation $\mathcal{E}_{\varepsilon}^{+}$is

$$
\begin{aligned}
& \tilde{u}_{t}=\tilde{v}_{x}+\varepsilon^{3} \cdot\left(\tilde{u}_{x} \tilde{u}_{x x}+\tilde{u}_{x x} \tilde{v}+\tilde{u}_{x} \tilde{v}_{x}+\tilde{v} \tilde{v}_{x}\right), \\
& \tilde{v}_{t}=\tilde{u}_{x x x}+\tilde{u} \tilde{u}_{x}-\varepsilon^{3} \cdot\left(\tilde{u}_{x x}^{2}+\tilde{u}_{x} \tilde{u}_{x x x}+\tilde{u}_{x x x} \tilde{v}+2 \tilde{u}_{x x} \tilde{v}_{x}+\tilde{u}_{x} \tilde{v}_{x x}+\tilde{v}_{x}^{2}+\tilde{v} \tilde{v}_{x x}\right) .
\end{aligned}
$$

The Miura transformation from $\mathcal{E}_{\varepsilon}^{+}$to (2) is

$$
\begin{aligned}
u= & \tilde{u}-2 \varepsilon \tilde{u}_{x}+2 \varepsilon^{2} \cdot\left(\tilde{u}_{x x}+\tilde{v}_{x}\right)+\varepsilon^{3} \cdot\left(\tilde{u} \tilde{v}+\tilde{u} \tilde{u}_{x}\right) \\
v= & \tilde{v}-2 \varepsilon \tilde{v}_{x}+2 \varepsilon^{2} \cdot\left(\tilde{v}_{x x}+\tilde{u}_{x x x}+\tilde{u} \tilde{u}_{x}\right)+\varepsilon^{3} \cdot\left(\frac{1}{3} \tilde{u}^{3}+\tilde{v}^{2}+\tilde{u} \tilde{u}_{x x}+\tilde{v} \tilde{v}_{x}+\tilde{u}_{x} \tilde{v}\right) \\
& \quad-2 \varepsilon^{4} \cdot\left(\tilde{u}_{x} \tilde{u}_{x x}+\tilde{u}_{x} \tilde{v}_{x}+\tilde{u}_{x x} \tilde{v}+\tilde{v} \tilde{v}_{x}\right)+\varepsilon^{6} \cdot\left(\frac{1}{3} \tilde{v}^{3}+\frac{1}{3} \tilde{u}_{x}^{3}+\tilde{u}_{x}^{2} \tilde{v}+\tilde{u}_{x} \tilde{v}^{2}\right) .
\end{aligned}
$$

The deformed equation $\mathcal{E}_{\varepsilon}^{-}$is

$$
\begin{aligned}
& \tilde{u}_{t}=\tilde{v}_{x}+\varepsilon^{3} \cdot\left(\tilde{u}_{x} \tilde{u}_{x x}-\tilde{u}_{x x} \tilde{v}-\tilde{u}_{x} \tilde{v}_{x}+\tilde{v} \tilde{v}_{x}\right) \\
& \tilde{v}_{t}=\tilde{u}_{x x x}+\tilde{u} \tilde{u}_{x}+\varepsilon^{3} \cdot\left(\tilde{u}_{x x}^{2}+\tilde{u}_{x} \tilde{u}_{x x x}-\tilde{u}_{x x x} \tilde{v}-2 \tilde{u}_{x x} \tilde{v}_{x}-\tilde{u}_{x} \tilde{v}_{x x}+\tilde{v}_{x}^{2}+\tilde{v} \tilde{v}_{x x}\right) .
\end{aligned}
$$


The Miura transformation from $\mathcal{E}_{\varepsilon}^{-}$to (2) is given through

$$
\begin{aligned}
u=\tilde{u}+2 \varepsilon \tilde{u}_{x}+2 \varepsilon^{2} \cdot\left(\tilde{u}_{x x}-\tilde{v}_{x}\right)+\varepsilon^{3} \cdot\left(\tilde{u} \tilde{v}-\tilde{u} \tilde{u}_{x}\right), \\
v=\tilde{v}+2 \varepsilon \tilde{v}_{x}+2 \varepsilon^{2} \cdot\left(\tilde{v}_{x x}-\tilde{u}_{x x x}-\tilde{u} \tilde{u}_{x}\right)+\varepsilon^{3} \cdot\left(\frac{1}{3} \tilde{u}^{3}+\tilde{v}^{2}+\tilde{u} \tilde{u}_{x x}-\tilde{v} \tilde{v}_{x}-\tilde{u}_{x} \tilde{v}\right) \\
\quad+2 \varepsilon^{4} \cdot\left(\tilde{u}_{x} \tilde{u}_{x x}-\tilde{u}_{x} \tilde{v}_{x}-\tilde{u}_{x x} \tilde{v}+\tilde{v} \tilde{v}_{x}\right)+\varepsilon^{6} \cdot\left(\frac{1}{3} \tilde{v}^{3}-\frac{1}{3} \tilde{u}_{x}^{3}+\tilde{u}_{x}^{2} \tilde{v}-\tilde{u}_{x} \tilde{v}^{2}\right) .
\end{aligned}
$$

Extended equations (4) and (5) consist of the original Boussinesq flows and the adjoint flows at $\varepsilon^{3}$, which will be further discussed in more detail. The Poisson structure (3) for the extended equations $\mathcal{E}_{\varepsilon}^{ \pm}$together with the Miura transformations $\mathcal{E}_{\varepsilon}^{ \pm} \rightarrow \mathcal{E}_{0}$ induce [8] the Poisson structures $\hat{A}_{1} \mp \varepsilon^{3} \hat{A}_{2}$ for Boussinesq's equation (21), here $\hat{A}_{1}$ is given through (3) and

$$
\hat{A}_{2}=\left(\begin{array}{cc}
8 D_{x}^{3}+u D_{x}+D_{x} \circ u & 3 v D_{x}+v_{x} \\
3 v D_{x}+2 v_{x} & D_{x}^{5}+5 \cdot\left(u D_{x}^{3}+D_{x}^{3} \circ u\right)- \\
& -3 \cdot\left(u_{x x} D_{x}+D_{x} \circ u_{x x}\right)+u D_{x} \circ u
\end{array}\right)
$$

are its first and second structures, respectively (see [9] and references therein). The Hamiltonians for the extensions $\mathcal{E}_{\varepsilon}^{ \pm}$are inherited from the original functionals, which are described in Proposition 1 below, by using the Miura substitutions.

Proposition 1. The densities of Hamiltonian functionals for the Boussinesq equation can be obtained using two different recurrence relations, which are

$$
\begin{aligned}
& \tilde{u}_{0}=u, \quad \tilde{v}_{0}=v, \quad \tilde{u}_{1}= \pm 2 u_{x}, \\
& \tilde{v}_{1}= \pm 2 v_{x}, \quad \tilde{u}_{2}=2 u_{x x} \mp 2 v_{x}, \quad \tilde{v}_{2}=2 v_{x x} \mp 2 u_{x x x} \mp 2 u u_{x} \text {, } \\
& \tilde{u}_{n}= \pm 2 D_{x}\left(\tilde{u}_{n-1}\right)-2 D_{x}^{2}\left(\tilde{u}_{n-2}\right) \mp 2 D_{x}\left(\tilde{v}_{n-2}\right)+\sum_{k+\ell=n-3}\left[-\tilde{u}_{k} \tilde{v}_{\ell} \mp \tilde{u}_{k} D_{x}\left(\tilde{u}_{\ell}\right)\right] \text {, } \\
& n \geq 3 \\
& \tilde{v}_{3}= \pm 2 D_{x}\left(\tilde{v}_{2}\right) \mp 2 D_{x}^{3}\left(\tilde{u}_{1}\right)-2 D_{x}^{2}\left(\tilde{v}_{1}\right) \mp\left[u D_{x}\left(\tilde{u}_{1}\right)+\tilde{u}_{1} u_{x}\right] \\
& -\frac{1}{3} u^{3}-v^{2}-u u_{x x} \mp u v_{x} \mp u_{x} v \\
& \tilde{v}_{n}= \pm 2 D_{x}\left(\tilde{v}_{n-1}\right) \mp 2 D_{x}^{3}\left(\tilde{u}_{n-2}\right)-2 D_{x}^{2}\left(\tilde{v}_{n-2}\right) \\
& \mp \sum_{k+\ell=n-2} 2 \tilde{u}_{k} D_{x}\left(\tilde{u}_{\ell}\right)-\sum_{k+\ell+m=n-3} \frac{1}{3} \tilde{u}_{k} \tilde{u}_{\ell} \tilde{u}_{m} \\
& +\sum_{k+\ell=n-3}\left[-\tilde{v}_{k} \tilde{v}_{\ell}-\tilde{u}_{k} D_{x}^{2}\left(\tilde{u}_{\ell}\right) \mp \tilde{u}_{k} D_{x}\left(\tilde{v}_{\ell}\right) \mp D_{x}\left(\tilde{u}_{k}\right) \tilde{v}_{\ell}\right] \\
& +\sum_{k+\ell=n-4} 2 \cdot\left[ \pm D_{x}\left(\tilde{u}_{k}\right) D_{x}^{2}\left(\tilde{u}_{\ell}\right)+D_{x}\left(\tilde{u}_{k}\right) D_{x}\left(\tilde{v}_{\ell}\right)+D_{x}^{2}\left(\tilde{u}_{k}\right) \tilde{v}_{\ell} \pm \tilde{v}_{k} D_{x}\left(\tilde{v}_{\ell}\right)\right] \text {, } \\
& n=4,5 \text {, }
\end{aligned}
$$




$$
\begin{aligned}
\tilde{v}_{n}= \pm 2 D_{x}\left(\tilde{v}_{n-1}\right) \mp 2 D_{x}^{3}\left(\tilde{u}_{n-2}\right)-2 D_{x}^{2}\left(\tilde{v}_{n-2}\right) & \\
& \mp \sum_{k+\ell=n-2} 2 \tilde{u}_{k} D_{x}\left(\tilde{u}_{\ell}\right)-\sum_{k+\ell+m=n-3} \frac{1}{3} \tilde{u}_{k} \tilde{u}_{\ell} \tilde{u}_{m} \\
& +\sum_{k+\ell=n-3}\left[-\tilde{v}_{k} \tilde{v}_{\ell}-\tilde{u}_{k} D_{x}^{2}\left(\tilde{u}_{\ell}\right) \mp \tilde{u}_{k} D_{x}\left(\tilde{v}_{\ell}\right) \mp D_{x}\left(\tilde{u}_{k}\right) \tilde{v}_{\ell}\right] \\
& +\sum_{k+\ell=n-4} 2 \cdot\left[ \pm D_{x}\left(\tilde{u}_{k}\right) D_{x}^{2}\left(\tilde{u}_{\ell}\right)+D_{x}\left(\tilde{u}_{k}\right) D_{x}\left(\tilde{v}_{\ell}\right)+D_{x}^{2}\left(\tilde{u}_{k}\right) \tilde{v}_{\ell} \pm \tilde{v}_{k} D_{x}\left(\tilde{v}_{\ell}\right)\right] \\
& +\sum_{k+\ell+m=n-6}\left[-\frac{1}{3} \tilde{v}_{k} \tilde{v}_{l} \tilde{v}_{m} \mp \frac{1}{3} D_{x}\left(\tilde{u}_{k}\right) D_{x}\left(\tilde{u}_{\ell}\right) D_{x}\left(\tilde{u}_{m}\right)\right. \\
& \left.\quad-D_{x}\left(\tilde{u}_{k}\right) D_{x}\left(\tilde{u}_{\ell}\right) \tilde{v}_{m}+D_{x}\left(\tilde{u}_{k}\right) \tilde{v}_{\ell} \tilde{v}_{m}\right], \quad n \geq 6 .
\end{aligned}
$$

The ambiguity of signs of the differential terms does not affect the nontrivial conserved densities $\tilde{u}_{3 k}$ and $\tilde{v}_{3 k}$. The densities with subscripts $3 k+1,3 k+2$ are trivial for all $k \geq 0$.

The recurrence relations listed in Proposition 1 are also valid for the Hamiltonians of the modified Boussinesq equation [9] which are obtained using the Miura substitutions to (2). The modified fields themselves do not start these sequences of conserved densities and thus cannot be regarded as their negative terms $\tilde{u}_{-3}, \tilde{v}_{-3}$.

Further, consider the flow at $\varepsilon^{3}$ in the r.h.s. of equation (5); the second case related to (4) is analogous. We thus obtain the system

$$
\begin{aligned}
& u_{\tau}=v v_{x}+u_{x} u_{x x}-u_{x x} v-u_{x} v_{x}, \\
& v_{\tau}=v v_{x x}+v_{x}^{2}-u_{x} v_{x x}-2 u_{x x} v_{x}-u_{x x x} v+u_{x} u_{x x x}+u_{x x}^{2} .
\end{aligned}
$$

It is Hamiltonian w.r.t. the structure (3) and the functional with density

$$
\bar{H}=\frac{1}{6} v^{3}+\frac{1}{2} u_{x}^{2} v-\frac{1}{2} u_{x} v^{2}-\frac{1}{6} u_{x}^{3} .
$$

Note that system (6) is homogeneous w.r.t. the set of weights $|u|=2,|v|=3,|\tau|=-5$, $|x| \equiv-1$. We claim that equation (6) admits two infinite sequences of Hamiltonian symmetries $\left(u_{s}, v_{s}\right)=\varphi_{[s]}$ for all weights $|s|=-(6 k+3 \pm 2), k \in \mathbb{N}_{\geq 0}$. These flows reproduce (with proper modifications) the scheme for the symmetries of the original Boussinesq hierarchy. Namely, they are arranged according to the diagram

$$
\varphi_{[1]} \rightarrow \varphi_{[7]} \rightarrow \varphi_{[13]} \rightarrow \cdots, \quad \varphi_{[5]} \rightarrow \varphi_{[11]} \rightarrow \cdots .
$$

The flow $\varphi_{[5]}$ is the right-hand side of the adjoint Boussinesq equation (6) itself. The two sequences of Hamiltonians for the higher symmetries of (6) are obtained from the functionals for system (15) by separating the coefficients of the highest powers of $\varepsilon$.

Example 1. The components $\varphi_{[7]}^{1,2}$ of the symmetry $\varphi_{[7]}$ are

$$
\begin{gathered}
\varphi_{[7]}^{1}=v v_{x x x}-u_{x} v_{x x x}+2 v_{x} v_{x x}-2 u_{x x} v_{x x}-2 u_{x x x} v_{x}-u_{4 x} v+u_{x} u_{4 x}+2 u_{x x} u_{x x x} \\
\varphi_{[7]}^{2}=v v_{4 x}-u_{x} v_{4 x}+3 v_{x} v_{x x x}-3 u_{x x} v_{x x x}+2 v_{x x}^{2}-4 u_{x x x} v_{x x}-3 u_{4 x} v_{x}-u_{5 x} v \\
\quad+u_{x} u_{5 x}+3 u_{x x} u_{4 x}+2 u_{x x x}^{2} .
\end{gathered}
$$


The symmetry $\varphi_{[11]}$ that succeeds the equation in the hierarchy has the components

$$
\begin{gathered}
\varphi_{[11]}^{1}=v v_{7 x}-u_{x} v_{7 x}+4 v_{x} v_{6 x}-4 u_{x x} v_{6 x}+10 v_{x x} v_{5 x}-10 u_{x x x} v_{5 x}+16 v_{x x x} v_{4 x} \\
-16 u_{4 x} v_{4 x}-16 u_{5 x} v_{x x x}-10 u_{6 x} v_{2 x}-4 u_{7 x} v_{x}-u_{8 x} v+u_{x} u_{8 x} \\
+4 u_{x x} u_{7 x}+10 u_{x x x} u_{6 x}+16 u_{4 x} u_{5 x}, \\
\varphi_{[11]=v v_{8 x}^{2}}-u_{x} v_{8 x}+5 v_{x} v_{7 x}-5 u_{x x} v_{7 x}+14 v_{x x} v_{6 x}-14 u_{x x x} v_{6 x}+26 v_{x x x} v_{5 x} \\
-26 u_{4 x} v_{5 x}+16 v_{4 x}^{2}-32 u_{5 x} v_{4 x}-26 u_{6 x} v_{x x x}-14 u_{7 x} v_{x x}-5 u_{8 x} v_{x} \\
-u_{9 x} v+u_{x} u_{9 x}+5 u_{x x} u_{8 x}+14 u_{3 x} u_{7 x}+26 u_{4 x} u_{6 x}+16 u_{5 x}^{2} . \\
\text { 2. THE KAUP-BOUSSINESQ EQUATION }
\end{gathered}
$$

\section{The KAUP-Boussinesq EQUATION}

Now we construct the Gardner deformation for the Kaup-Boussinesq equation

$$
u_{t}=u u_{x}+v_{x}, \quad v_{t}=(u v)_{x}+u_{x x x} .
$$

Theorem 2. The integrable extension $\mathcal{E}_{\varepsilon}$ of the Kaup-Boussinesq equation (7) is the system

$$
\begin{aligned}
& \tilde{u}_{t}=\tilde{u} \tilde{u}_{x}+\tilde{v}_{x}+\varepsilon \cdot\left(\tilde{u} \tilde{u}_{x x}+\tilde{u}_{x}^{2}+(\tilde{u} \tilde{v})_{x}\right), \\
& \tilde{v}_{t}=(\tilde{u} \tilde{v})_{x}+\tilde{u}_{x x x}-\varepsilon \cdot\left(2 \tilde{u}_{x} \tilde{u}_{x x}+\tilde{u} \tilde{u}_{x x x}+\tilde{u}_{x} \tilde{v}_{x}+\tilde{u} \tilde{v}_{x x}-\tilde{v} \tilde{v}_{x}\right) .
\end{aligned}
$$

System (8) is Hamiltonian w.r.t. the structure (3) and the functional

$$
\mathcal{H}(\varepsilon)=\int\left(\frac{1}{2} \tilde{u}^{2} \tilde{v}+\frac{1}{2} \tilde{v}^{2}-\frac{1}{2} \tilde{u}_{x}^{2}+\frac{1}{2} \varepsilon \cdot\left[\tilde{u} \tilde{u}_{x}^{2}+2 \tilde{u} \tilde{u}_{x} \tilde{v}+\tilde{u} \tilde{v}^{2}\right]\right) \mathrm{d} x .
$$

The Miura transformation $\mathcal{E}_{\varepsilon} \rightarrow \mathcal{E}_{0}$ is given through

$$
u=\tilde{u}+\varepsilon \cdot\left(\tilde{u}_{x}+\tilde{v}\right), \quad v=\tilde{v}+\varepsilon \cdot\left(\tilde{u} \tilde{u}_{x}+\tilde{u}_{x x}+\tilde{u} \tilde{v}+\tilde{v}_{x}\right) .
$$

The recurrence relations upon densities of the Hamiltonian functionals for (7) are

$$
\begin{aligned}
& \tilde{u}_{0}=u, \quad \tilde{v}_{0}=v, \quad \tilde{u}_{k}=-D_{x}\left(\tilde{u}_{k-1}\right)-\tilde{v}_{k-1}, \\
& \tilde{v}_{k}=-D_{x}^{2}\left(\tilde{u}_{k-1}\right)-D_{x}\left(\tilde{v}_{k-1}\right)-\sum_{\ell+m=k-1}\left[\tilde{u}_{\ell} D_{x}\left(\tilde{u}_{m}\right)+\tilde{u}_{\ell} \tilde{v}_{m}\right], \quad k>0 .
\end{aligned}
$$

Relations (10) do not produce any auxiliary trivial conserved densities.

Relations (10) provide the formulas for the Hamiltonians of the (twice- and thrice-) modified Kaup-Boussinesq equations [10]. The modified fields are conserved; nevertheless, we note that they are not obtained from the Hamiltonians for (17) by the Miura substitutions and that the modified fields cannot be used as the negative terms in the general scheme of (10).

Substitution (9) provides the canonical factorization of the trivially extended Poisson structure $\hat{A}_{1}+2 \varepsilon \hat{A}_{2}$ for the Kaup-Boussinesq equation, where $\hat{A}_{1}$ is defined in (3) and

$$
\hat{A}_{2}=\left(\begin{array}{cc}
D_{x} & \frac{1}{2} D_{x} \circ u \\
\frac{1}{2} u D_{x} & D_{x}^{3}+\frac{1}{2} D_{x} \circ v+\frac{1}{2} v D_{x}
\end{array}\right) .
$$

The two sequences of Hamiltonians, see (10), for the deformed Kaup-Boussinesq equation (8) are inherited from the original functionals for the Kaup-Boussinesq equation (77) by using the Miura substitution (9). The correlation between the higher flows for (8) and the symmetries of (7) is standard, see [3] and references therein. 
Taking the flow at $\varepsilon$ in extension (8), we obtain the adjoint Kaup-Boussinesq equation

$$
\begin{aligned}
& u_{\tau}=u u_{x x}+u_{x}^{2}+u_{x} v+u v_{x}, \\
& v_{\tau}=-\left(2 u_{x} u_{x x}+u u_{x x x}+u_{x} v_{x}+u v_{x x}-v v_{x}\right) .
\end{aligned}
$$

System (11) is bi-Hamiltonian w.r.t. two compatible local Poisson structures $\Gamma_{1}$ and $\Gamma_{2}$, where $\Gamma_{1}$ is (3) and

$$
\Gamma_{2}=\left(\begin{array}{cc}
0 & D_{x} \circ u_{x}+D_{x} \circ v \\
u_{x} D_{x}+v D_{x} & -u_{x x} D_{x}-D_{x} \circ u_{x x}- \\
& -v_{x} D_{x}-D_{x} \circ v_{x}
\end{array}\right)
$$

We conclude that the adjoint Kaup-Boussinesq equation (11) is completely integrable.

Remark 1. Both Poisson structures (3) and (12) for (11) are of differential order 1. This indicates that the system can be further extended with a higher order symbol such that its complete integrability is preserved. The situation is analogous to the Gardner extension of the Korteweg-de Vries equation [1; we recall that the extension of $\mathrm{KdV}$ resulted in the dispersionless modified $\mathrm{KdV}$ equation whose Poisson structures are of order 1 .

Remark 2. The 'minus' Kaup-Boussinesq equation $u_{t}=u u_{x}+v_{x}, v_{t}=(u v)_{x}-u_{x x x}$ admits a unique real quadratic extension

$$
\begin{aligned}
& \tilde{u}_{t}=\tilde{u} \tilde{u}_{x}+\tilde{v}_{x}+\varepsilon \cdot\left(\tilde{u} \tilde{u}_{x x}+\tilde{u}_{x}^{2}\right), \\
& \tilde{v}_{t}=(\tilde{u} \tilde{v})_{x}-\tilde{u}_{x x x}-\varepsilon \cdot\left(\tilde{u}_{x} \tilde{v}_{x}+\tilde{u} \tilde{v}_{x x}\right)-\varepsilon^{2} \cdot\left(\tilde{u}^{2} \tilde{u}_{x x x}+\tilde{u} \tilde{u}_{x} \tilde{u}_{x x}+\tilde{u}_{x}^{3}\right) .
\end{aligned}
$$

System (13) is assinged by the first structure (3) to the Hamiltonian

$$
\mathcal{H}^{\prime}(\varepsilon)=\int\left(\frac{1}{2} \tilde{u}^{2} \tilde{v}+\frac{1}{2} \tilde{v}^{2}+\frac{1}{2} \tilde{u}_{x}^{2}+\varepsilon \cdot \tilde{u} \tilde{u}_{x} \tilde{v}+\frac{1}{2} \varepsilon^{2} \cdot \tilde{u}^{2} \tilde{u}_{x}^{2}\right) \mathrm{d} x .
$$

The invertible Miura transformation from the deformed equation to (17) is

$$
\left\{u=\tilde{u}, v=\tilde{v}+\varepsilon \tilde{u} \tilde{u}_{x}\right\} \quad \Longleftrightarrow \quad\left\{\tilde{u}=u, \tilde{v}=v-\varepsilon u u_{x}\right\} .
$$

The recurrence relation obtained from (14) provides only the Casimirs $\int u \mathrm{~d} x$ and $\int v \mathrm{~d} x$; all the conserved densities $\tilde{v}_{k}$ are trivial if $k>0$. Therefore deformation (13 14) of the 'minus' Kaup-Boussinesq equation is trivial.

Acknowledgements. The authors thank E. V.Ferapontov, B. A. Kupershmidt, and A. V. Mikhailov for helpful discussions and are also grateful to M. Brodovsky for computer programming. The content of this paper was reported at the 5th Workshop on Quantization, Dualities, and Integrable Systems at Pamukkale University. The research was partially supported by the Scientific and Technological Research Council of Turkey (TUBITAK). A part of this research was carried out while A. V.K. was visiting at Middle East Technical University (Ankara).

\section{REFERENCES}

[1] Miura R M, Gardner C S, and Kruskal M D 1968 Korteweg-de Vries equation and generalizations. II. Existence of conservation laws and constants of motion J. Math. Phys. 9 1204-1209. 
[2] Kupershmidt B A 1982 On algebraic models of dynamical systems Lett. Math. Phys. 6 no.2, 85-89.

[3] Kupershmidt B A 1983 Deformations of integrable systems Proc. Roy. Irish Acad. A83 no.1, 45-74.

[4] Fordy A P 1983 Projective representations and deformations of integrable systems Proc. Roy. Irish Acad. A83 no.1, 75-93.

[5] Mathieu P 1988 Supersymmetric extension of the Korteweg-de Vries equation J. Math. Phys. 29 no.11, 2499-2506.

[6] Andrea S, Restuccia A, and Sotomayor A 2005 The Gardner category and non-local conservation laws for $N=1$ super KdV J. Math. Phys. 46 no.10, 103517, 11 p.

[7] Labelle $P$ and Mathieu $P 1991$ A new $N=2$ supersymmetric Korteweg-de Vries equation J. Math. Phys. 32 no.4, 923-927.

[8] Manin Yu I 1978 Algebraic aspects of non-linear differential equations Itogi Nauki $i$ Tekhniki. Ser. Sovremennye Prob. Mat. 11 5-151; transl. in J. Sov. Math. 11 $1-122$.

[9] Pavlov M V 2004 The Boussinesq equation and Miura-type transformations, Fundam. Prikl. Mat. 10 no.1, 175-182.

[10] Pavlov $M V 2002$ Integrable systems and metrics of constant curvature, J. Nonlin. Math. Phys. 9 suppl.1, 173-191.

[11] Bocharov A V, Chetverikov V N, Duzhin S V et al 1999 Symmetries and Conservation Laws for Differential Equations of Mathematical Physics AMS Providence RI. Krasil'shchik I and Vinogradov A (eds).

[12] Igonin S and Krasil'shchik I S 2003 On one-parametric families of Bäcklund transformations Adv. Stud. Pure Math. 37 99-114.

[13] Kiselev A V 2002 On Bäcklund autotransformation for the Liouville equation Vestnik Moskovskogo Univ. 6 22-26.

[14] Kiselev $A V$ and Golovko $V A 2004$ On non-abelian coverings over the Liouville equation Acta Appl. Math. 83 no.1-2, 25-37.

[15] Kiselev $A \quad V$ and Wolf $T 2006$ Supersymmetric representations and integrable fermionic extensions of the Burgers and Boussinesq equations Symmetry, Integrability and Geometry: Methods and Applications (SIGMA) 2 no.030, 19 p.

Department of Physics, Middle East Technical University, 06531 Ankara, Turkey.

E-mail address: karasu@metu.edu.tr

Permanent address (A. V.K.): Department of Higher Mathematics, Ivanovo State Power University, Rabfakovskaya str. 34, Ivanovo, 153003 Russia.

Current address: Department of Physics, Middle East Technical University, 06531 Ankara, Turkey.

E-mail address: arthemy@newton.physics.metu.edu.tr 